\title{
Smart Parking System Based on an Ultrasonic Sensor and Bluetooth Low Energy in the Internet of Things
}

\author{
Soobin Jeon and Dongmahn Seo ${ }^{+}$ \\ School of Information Technology Engineering, Daegu Catholic University, 13-13 \\ Hayangwo, Gyoungsansi Gyoungbuk 38430, Republic of Korea \\ sarum@cu.ac.kr
}

\begin{abstract}
Technology related to the Internet of Things (IoT) has contributed greatly to smart living environments. The smart parking system is one of the main services based on IoT applications. To identify parked vehicles, previous parking systems used special identification devices where Radio-Frequency Identification (RFID) tags were carried by the users and high-quality cameras could recognize the vehicle license numbers. However, the previous methods were inefficient in terms of cost and they were not user-friendly. Thus, to address these problems, we propose a smart parking system based on ultrasonic sensors and the received signal strength indicator (RSSI) in Bluetooth communication. The proposed system determines the available spaces by using sensor motes located in the parking spaces. In addition, it recognizes the location of a parked vehicle based on Bluetooth low energy (BLE) communication between the user's smartphone and the sensor motes. Furthermore, to improve the location awareness for users, we transform the RSSI strength into a distance range by applying the triangulation method. Based on IoT technology, the smart parking system can guide users to locations for their vehicles. We implemented the sensor motes for the ultrasonic sensor as well as the BLE modules in order to evaluate the proposed smart parking system. The sensor motes were deployed in parking spaces and we confirmed that the ultrasonic sensors successfully detected the available parking spaces, and they guided cars to the parking spaces divided into cell units.
\end{abstract}

Keywords: Bluetooth low energy, Internet of Things, location awareness, received signal strength indicator, smart parking system, ultrasonic sensor

\section{Introduction}

Vehicles are important modes of transportation at present. However, the number of vehicles has increased greatly in recent years and the limited availability of vehicle parking spaces has caused several problems. Recently, parking management systems have been developed where electronic displays provide information about 
the available parking spaces as well as other types of parking information. However, this approach is not convenient because drivers must search for information about the available parking spaces via the electronic display and then search directly for the locations of empty parking spaces. Thus, despite the high installation costs for electronic displays, the current parking management systems are not satisfactory because they do not provide simple and useful information.

In the era of the Internet of Things (IoT), smart "things" can be embedded with various types of sensor devices and communication devices, which may be deployed in different environments and the network can be configured without human interference, where their interactions with each other make new information available in the IoT ecosystem. In the future, it is expected that many application services well be developed for IoT environments because the sensors and microprocessors will have better performance, as well as being smaller in size with lower costs. Among the many expected IoT services, smart parking services are particularly important, and they may combine IoT technology with existing vehicle parking systems (Gilbert et al. 2019; Jin et al. 2014).

In this study, we designed and implemented a smart parking system based on ultrasonic sensors and Bluetooth low energy (BLE) devices embedded in wireless parking sensor motes. The system can monitor the empty parking spaces and also identify the locations where individual users have parked their vehicles. In addition, this system can provide a location awareness function in real-time for users, thereby guiding them to the locations of the parking spaces and their parked vehicles. In experiments, we showed that the ultrasonic sensors could successfully manage the available parking spaces.

The location awareness service is based on the received signal strength indicator (RSSI) for a BLE module, where we employ an equation to transform the RSSI strength into the distance. Using three distances between the sensor motes and the user, triangulation is employed to support the location awareness function. In experiments, we evaluated the range of error between the estimated distances and real measured values. We divided the parking spaces into cell units to facilitate the location guidance service. As the user moves toward the parking space, tracking is performed based on the triangulation method with the three estimated distance values. In our experiments, we showed that the location guidance service based on RSSI strength is appropriate for finding parking spaces.

The remainder of this paper is organized as follows. In Section 2, we describe related research into existing parking systems and location awareness technologies based on wireless communication. In Section 3, we explain our proposed smart parking system based on ultrasonic sensors and Bluetooth communication with wireless parking sensor motes. In Section 4, we present the results obtained after implementing the proposed system and performance evaluations of the smart parking system. In Section 5, we give our conclusions and suggestions for future 
research.

\section{Related Work}

\subsection{Parking Management System}

RFID and camera devices are used to obtain information from vehicles in order to identify them in parking spaces. Thus, the parking management system uses an RFID device as a tag to identify a vehicle when it enters a car park as well as to guide users when they are searching for an empty parking space. In order to provide guidance to empty parking spaces, an RFID module must be attached to the vehicle and an additional display device is installed at the car park entrance (Wei et al., 2012). The user's smartphone can be employed to guide them to an empty parking space and improve usability for users. However, parking management systems that use RFID devices have high maintenance costs because this approach requires special RFID modules for users and their vehicles (Mainetti et al. 2014).

If a camera is used in the parking management system, it may mistakenly identify parked vehicles in 3-5 parking adjacent spaces. This approach recognizes the parked vehicles by taking pictures of the vehicle number plates (Lee et al., 2014). The recognition rate for vehicle number plates is improving due to progress in image processing and camera technology, but a camera can only cover a limited number of parking spaces and a high-quality camera is required to recognize the vehicle number plates. The installation and management costs will be high if the car park is large with many parking spaces.

\subsection{Estimation Locations with Wireless Communication}

Time of arrival (ToA), time difference of arrival (TDoA), RSSI, and angle of arrival (AoA) methods may be employed to estimate the locations of users. These methods exploit various characteristics related to the frequency of wireless communication. The ToA method is also known as the time of flight. ToA uses the transmission time of a radio signal from the sender to the receiver to estimate the distance. The speed of a radio signal is equal to that of light. If this method is employed to facilitate location awareness among users, then accurate time synchronization between the sender and reviver is essential. However, time synchronization among smart things incurs high overhead costs. Thus, many studies have investigated accurate time synchronization in the IoT (Sathyan et al. 2010; Kaune et al. 2011).

Similar to ToA, TDoA exploits the delivery time for signals to estimate the locations of users, where the distance between the sender and receiver is calculated based on the elapsed time required to deliver signals. TDoA also exploits the time differences between signals received by several receivers when their location is already known. AoA estimates the direction based on the angles of the received signals. Thus, several antennae should be installed with directional properties, where the angles of the received signals are measured by determining the time differences between the signals that arrive at each antenna. This method is 
employed for estimating the direction rather than the distance.

The RSSI is a numerical representation of the radio signal's strength, where the RSSI has a larger value when the distance between the sender and receiver is smaller. The locations users can be estimated from this feature. The recently developed communication modules in smartphones can support application programming interface(API) by obtaining the RSSI information from other BLE modules. In contrast to the methods mentioned above for estimating the distance, these APIs can be used easily in general applications. However, the RSSI is highly sensitive to the surrounding operating environment, such as walls or pillars, so it is necessary to perform several filtering procedures in order to accurately determine the locations of users (Zaruba et al. 2007; Han et al. 2011; Jung and Sim, 2010).

\subsection{Route Finding Algorithm}

The shortest path algorithm seeks the path with the minimal cost between two nodes on a graph. Many algorithms have been developed for this purpose. In particular, the $A^{*}$ algorithm is a best-first search algorithm, which performs a heuristic search of the nodes based on rankings. The implementation of this method is simple but it can find the shortest path in a small number of computations. In the A* algorithm, a visited node is set as a closed node and an unvisited node is set as an open node. The A* algorithm visits the open nodes continually until it reaches the goal node. In the node visiting procedure, this algorithm assigns each node with a fitness degree. The fitness (f) is derived using Equation (1):

$$
f=g+h
$$

where $\mathrm{g}$ (goal) is the cost of moving from the start node to the current node and $\mathrm{h}$ (heuristic) is the estimated cost of moving from the current node to the goal node. The real cost of moving from the current node to the goal node is not known, so a heuristic value is applied. The straight distance from the current node to the goal node is usually employed to calculate the heuristic cost. The algorithm operates as follows. First, $\mathrm{f}$ is calculated for the neighbor nodes of the start node. The neighbor node with the smallest $f$ is visited. Second, the $f$ values of the open neighbor nodes are calculated. Third, the open node with the smallest $\mathrm{f}$ is visited. The A* algorithm saves all the previous states before the next steps. These steps are repeated until the goal node is found. Finally, after the goal node has been visited, the total path is calculated by following the saved paths.

The $\mathrm{A}^{*}$ algorithm has been used in game development because it is the most efficient route finding method, where it performs better than Dijkstra's algorithm and the best-first search method. However, the A* algorithm must manage the open nodes and closed nodes to create the new route, so it requires an increasingly large amount of memory as the map size increases (Hart et al. 1968).

\section{Smart Parking System}

Figure 1 illustrates the smart parking system proposed in this study. The smart 
parking system comprises wireless parking sensor motes, a parking management server, and an application running on the user's smartphone. The wireless parking sensor motes are installed on the ceiling above a parking space. Each mote comprises an ultrasonic sensor module, BLE communication module, and control module. The ultrasonic sensor in the sensor mote can determine whether the parking space is occupied. The sensor mote transmits the state of the parking space to the server and the server manages the states of all the parking spaces in the car park. The BLE module in the sensor mote communicates with the smart parking user application running on the user's smartphone. This module receives the identification information for each parked vehicle. In addition, the BLE module operates as a beacon to facilitate location awareness for users of the parking space.

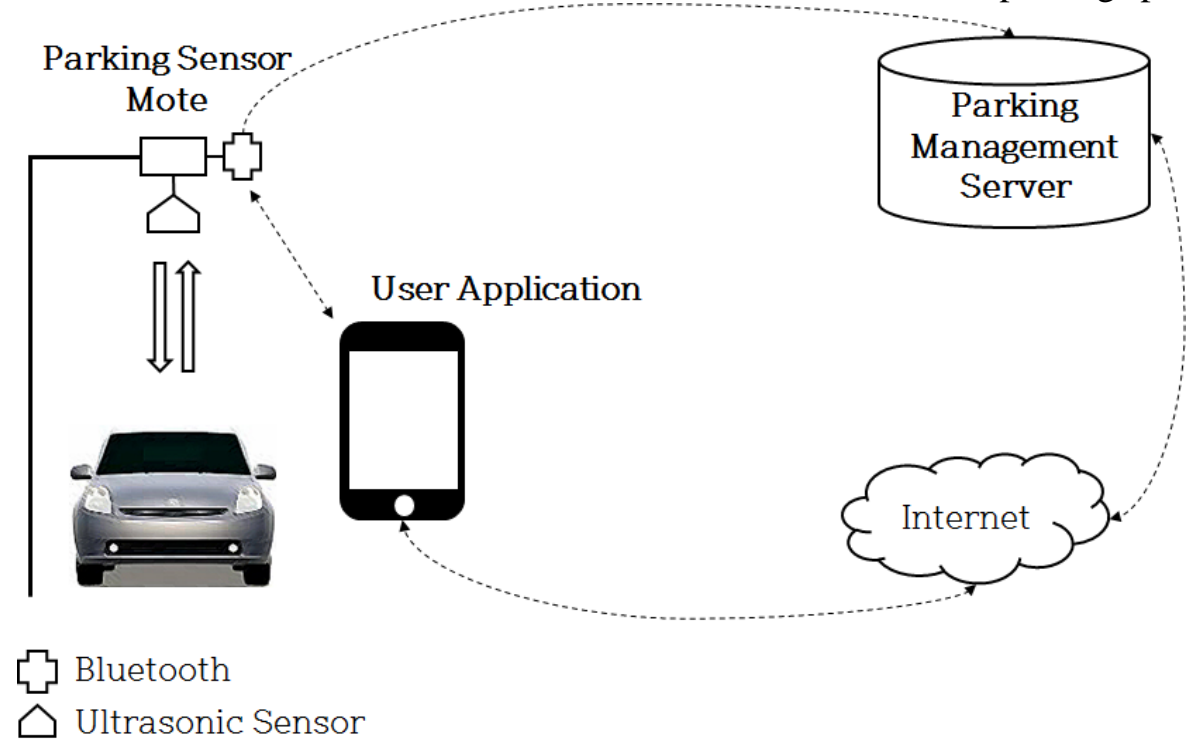

Fig. 1: Schematic illustrating the smart parking system.

The parking management server receives information from the sensor motes, including the occupation status of the parking spaces and the identifications of the parked vehicles. Using this information, the server can manage the real-time state of the parking spaces and it sends this information to the users' smartphone, i.e., the recent status of the parking spaces and the locations of specific parked vehicles.

The user application for smart parking is installed on the user's smartphone and it communicates with the parking management server to provide recent information about the parking spaces. In addition, the user application communicates with the parking sensor mote and it transmits the identification information for the parked vehicle. The application also provides a location guidance service to users of the parking space by cooperating with several sensor motes.

The steps in the operation of the smart parking system are as follows. First, 
when a vehicle enters a parking space, the ultrasonic sensor on the sensor mote recognizes whether a vehicle is or is not parked. Second, after the vehicle has been parked in the parking space, the user submits their parking location via the smart parking application on their smartphone. In this process, the user application communicates with the sensor mote assigned to the corresponding parking space. Third, the user sends their identification details (ID) to the sensor mote via Bluetooth. After the sensor mote receives the user's ID, it transfers the ID and information about the parking space to the parking management server. Fourth, the server manages the locations of the parked vehicles and information about car vehicle locations for users of the parking spaces. Thus, by employing the smart parking application on their smartphone, users can search for their vehicle's location and receive guidance to the location of the parking space.

\subsection{Vehicle Parking Decisions}

Ultrasonic sensors have been used previously for recognizing the presence of vehicles in parking spaces, and they have also been deployed on roads to measure the traffic volume and the presence of vehicles (Jo et al. 2014; Jeon et al. 2014). In our method, we used a wireless parking sensor mote with an ultrasonic sensor to check the status of parking spaces.

The steps involved in recognizing the presence of a vehicle in a parking space are as follows. First, an ultrasonic sensor is installed on the ceiling above a parking space and the distance from the ceiling to the ground is measured using a backward echo wave from the ultrasonic sensor. This distance is used as a basic value to determine the vehicle parking status. According to the enforcement regulations for indoor parking spaces, the height of parking spaces must be at least $2.3 \mathrm{~m}$ from the ceiling to the ground. In our method, we assume that the height is $2.5 \mathrm{~m}$. If the parking space is empty, the ultrasonic sensor attached to the ceiling detects a distance of $2.5 \mathrm{~m}$ from the floor. Thus, a distance of $2.5 \mathrm{~m}$ is used as a basic value to determine the parking status for parking spaces. If a vehicle is parked in the space, the sensor would detect a distance between $0.5 \mathrm{~m}$ and $1 \mathrm{~m}$ depending on the height of the vehicle. Therefore, sensor data values less than $2.5 \mathrm{~m}$ indicate that a vehicle is occupying the parking space.

\subsection{Location Awareness in Parking Space}

To provide a location awareness service related to parking spaces, a real-time awareness method is needed based on the movements of users. Recently, location awareness methods have been developed based on the inter-relationships between pervasive smart things deployed in the environment and this is an active research area (Mainetti et al. 2014; Lee et al. 2003; Zaruba et al. 2007). Our smart parking system provides a location awareness service to parking space users. After the vehicle has been parked, each user is distinguished according to their parking space because the parking application on the user's smartphone automatically transfers identification information to the sensor mote. 
Methods based on global positioning system(GPS) or Wifi have been employed for location awareness in existing IoT systems. However, GPS is not a suitable method for location awareness in an indoor parking environment. In addition, the Wifi method may be problematic when saving the parking locations and the parking location saving function is important in our system. In order to save their parking location, the smart parking system users must transfer their identification information to the sensor mote. However, smartphone users generally exploit a public access point (AP) or their potable AP for Wifi communication, so users must change their existing AP communication method to the sensor mote in the parking space in order to save their parking location. Therefore, the connection to the existing AP should be closed temporarily.

Recently, Bluetooth has been employed for communication in IoT services and it is accessible by most smartphones. The communication range of BLE is tens of meters so it is suitable for use in indoor parking spaces. In addition, the amount of energy consumed by BLE is low compared with the Wifi method and it can connect to other devices via a smartphone. However, in contrast to Wifi, BLE can connect with the sensor motes in parking spaces while other forms of communication are running, which is why we employ the BLE method for communicating with the sensor motes.

Two types of location awareness methods are implemented in our smart parking system. The first type involves saving the location of a parked vehicle. The location is usually saved near the parked vehicle. The range of user movements is small, so priority is given to support this process more accurately than the quick process. The second type involves support for real-time location guidance services for parking space users. Unlike the first type, the users are moving constantly to find a parking space for their vehicle, so the range of user movements is large. Thus, a quick location awareness process is necessary to provide real-time routing guidance.

In this study, in order to satisfy the requirements for accurate and quick location awareness regarding indoor parking spaces, we propose two types of location awareness methods, which exploit the RSSI in BLE. The RSSI represents the strength of the received signal, where the strength is greater when the distance is smaller, and vice versa.

\subsubsection{Location Awareness for Parked Vehicles}

In a smart parking space, the BLE modules of several sensor motes can communicate with the user's smartphone simultaneously. The RSSI strength from a sensor mote becomes stronger as the distance between the sensor mote and the user smartphone decreases. Thus, the sensor mote with the strongest RSSI strength is selected to save the location of a parked vehicle. There is no need to calculate accurate distances to the connected sensor motes to find the sensor mote for the currently parked vehicle. However, a highly reliable selection of the correct sensor 
mote can be achieved when the relative values of the RSSI strengths are compared.

To confirm the user's identity, the user application sends the Universal Subscriber Identification Module ID (USIM ID) of the smartphone to the selected sensor mote. The USIM ID has a unique value that identifies the user, which is supplied by the communication company, and it is possible to identify the user in the parking space based on this information.

To enhance reliability when recognizing the locations of parked vehicles, we use three sensor motes with high RSSI strengths as the candidate sensor motes related to the currently parked vehicle. Among the three candidates, the sensor mote with the highest RSSI strength is assigned to the currently parked vehicle.

We classify the state of each sensor mote according to three types. The first state is the "standby mode," which means that a vehicle is not present in the parking space. The second state is the "USIM ID receiving mode," which means that the ultrasonic sensor has recognized a parked vehicle and the sensor mote is waiting to receive the user's USIM ID. The final state is the "parked mode," which shows that the USIM ID has been received from the user and the location of the parked vehicle has been saved in the management server, and thus the parking process is complete. In an exceptional situation, if the user does not record the location of their parked vehicle for a long period of time, then we set the state of the corresponding parking space as "parked mode."

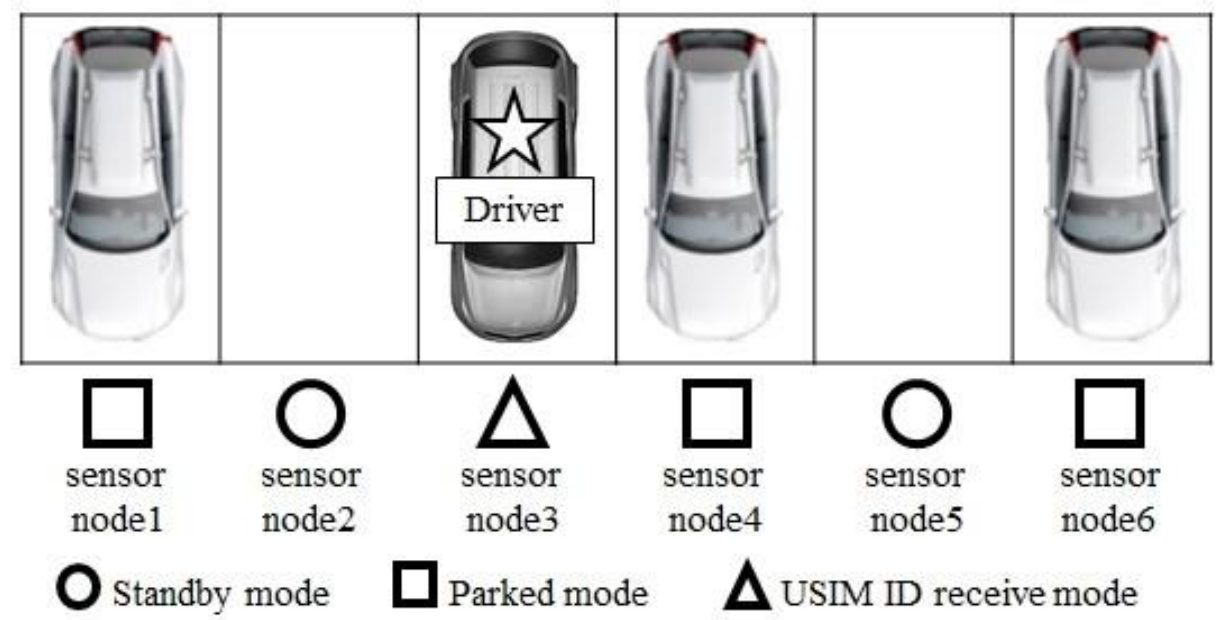

Fig. 2: Different states of a parking sensor mote.

If the sensor mote is in the "USIM ID receiving mode," this means that the correct sensor mote has been selected. Thus, the user's smartphone sends the USIM ID information to the selected sensor mote. However, if the selected sensor mote is not in the "USIM ID receiving mode, then the pairing operation must proceed with the candidate sensor mote that has the next highest RSSI strength.

Figure 2 shows the state of each sensor mote in a parking area with six parking 
spaces. In this example, a vehicle is parked in the parking space corresponding to sensor mote 3 and the ultrasonic sensor recognizes the parked vehicle. Sensor mote 3 changes its state to "USIM ID receiving mode" and it waits to connect with the user's smartphone for a certain period. The state of sensor mote 2 is "standby mode," so the incorrect choice would be made if the user selects sensor mote 2 arbitrarily. Thus, a pairing operation is executed to find the correct sensor mote. After pairing with sensor mote 3, it is confirmed that sensor mote 3 is in the "USIM ID receiving mode." Therefore, the location of the parked vehicle is in the area of sensor mote 3 . The state of sensor mote 3 then changes to "parked mode." Therefore, the paring operation can make a correction even if the user has made a mistake during the selection of the sensor mote.

\subsubsection{Location Awareness for Real-Time Location Guidance}

Our smart parking system provides a real-time location guide service to parking space users, where this service guides users to their parked vehicles or to parking spaces. This service requires a new real-time location awareness method because it must track the locations of users during the limited time when they are moving continuously in the car park.

As mentioned in the previous section, the RSSI strength of BLE is used to facilitate location awareness guidance to parked vehicles and parking spaces. However, the BLE strength cannot be used when vehicles or pedestrians are moving because the sensor motes are not deployed in all areas. Thus, an accurate location awareness method and real-time processing are needed in order to guide the current user to the location of their vehicle. In this method, we divide the parking area into cell units where the cell size indicates the space where a vehicle can be parked. We use these cells to recognize the current locations of users.

\begin{tabular}{|c|c|c|c|c|c|}
\hline 1 & 2 & 3 & 4 & 5 & 6 \\
\hline 7 & $\mathrm{~A}$ & $\mathrm{~B}$ & $\mathrm{C}$ & $\mathrm{D}$ & $\mathrm{E}$ \\
\hline 8 & 9 & 10 & 11 & 12 & 13 \\
\hline 14 & $\mathrm{~F}$ & $\mathrm{G}$ & $\mathrm{H}$ & $\mathrm{I}$ & $\mathrm{J}$ \\
\hline 15 & 16 & 17 & 18 & 19 & 20 \\
\hline $\mathrm{K}$ & $\mathrm{L}$ & $\mathrm{M}$ & $\mathrm{N}$ & $\mathrm{O}$ & $\mathrm{P}$ \\
\hline
\end{tabular}

Fig. 3: Parking area divided into cell units.

Figure 3 shows an example of a parking area divided into cell units, where gray represents the cells occupied by vehicles. In our smart parking model, a cell represents one parking space and the white parts are not parking spaces, but instead, they represent the routes for movements by vehicles or people. The steps in the 
location awareness process for users are following. First, the user's smartphone is employed to measure the RSSI transmitted from the BLE module of a sensor mote. As shown in Figure 3, the sensor motes in each cell unit $(\mathrm{A}, \mathrm{B}, \mathrm{C}, \ldots)$ generate an RSSI from their BLE modules. Second, the three sensor motes are selected that transmit the highest RSSI strength. Third, the three RSSI values are transformed into distances from the current user to the location of each sensor mote. Finally, based on these three distance values, triangulation is employed to recognize the current location of the user in the parking area.

\subsubsection{Distance Measurement Based on the RSSI Signal Strength}

Based on the RSSI signal strength, we can calculate the distance between the sensor mote and the user's smartphone. We used two devices in our experiments and Figure 4 shows the experimental environment employed for deriving the formula for the distance based on the RSSI signal strength. As shown in Figure 4, the BLE module of the sensor mote was fixed and the smartphone moved a distance of up to $5 \mathrm{~m}$. We measured the RSSI strength at $10-\mathrm{cm}$ intervals for $5 \mathrm{~m}$. The experiment was performed 10 times in the same environment.

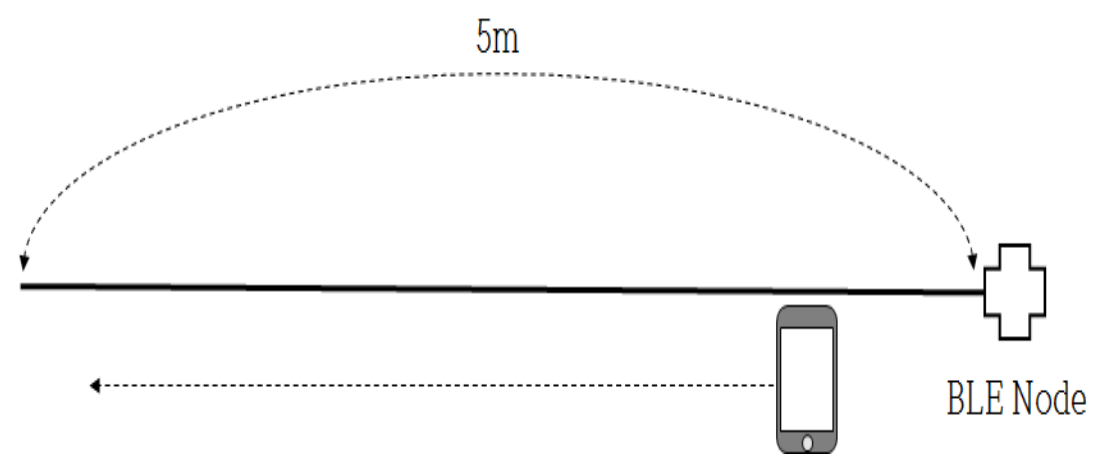

Fig. 4: Experimental environment for RSSI strength testing.

Figure 5 shows the average, minimum, and maximum values of the RSSI strength per $10 \mathrm{~cm}$ interval over $5 \mathrm{~m}$. To derive the formula for calculating the distance according to the RSSI strength, we plotted a trend line based on the average values. The distance equation (Equation (2)) was then derived from this trend line to transform the RSSI strength into the distance.

$$
\text { Distance }(m)=0.0035 e^{-0.006^{*} R B S I}
$$

The user's smartphone receives the RSSI of the BLE from the sensor motes in the parking space. The three sensor motes with the strongest RSSI strengths are then selected. Based on Equation (2), the RSSI strengths for each of the selected sensor motes are transformed into distances. The triangulation method is then employed to recognize the cell where the user is located based on the estimated distances. 


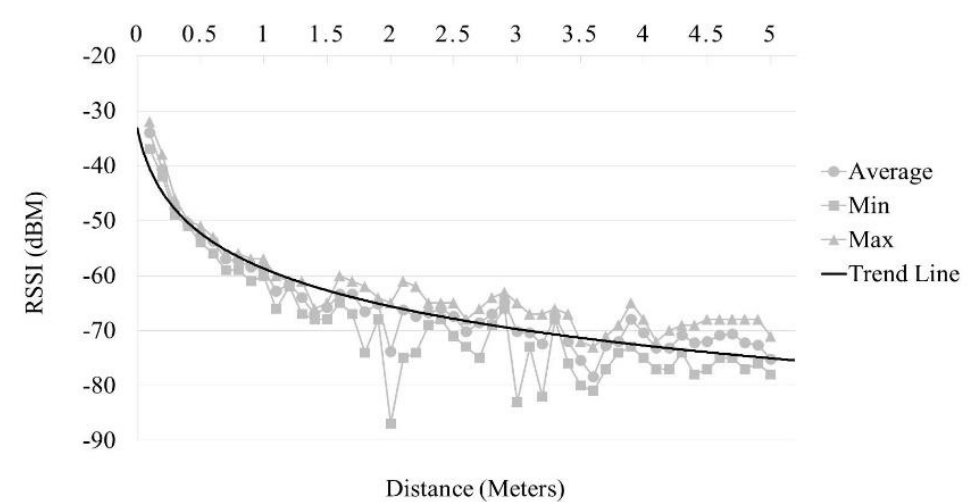

Fig. 5: RSSI strengths of BLE according to distance.

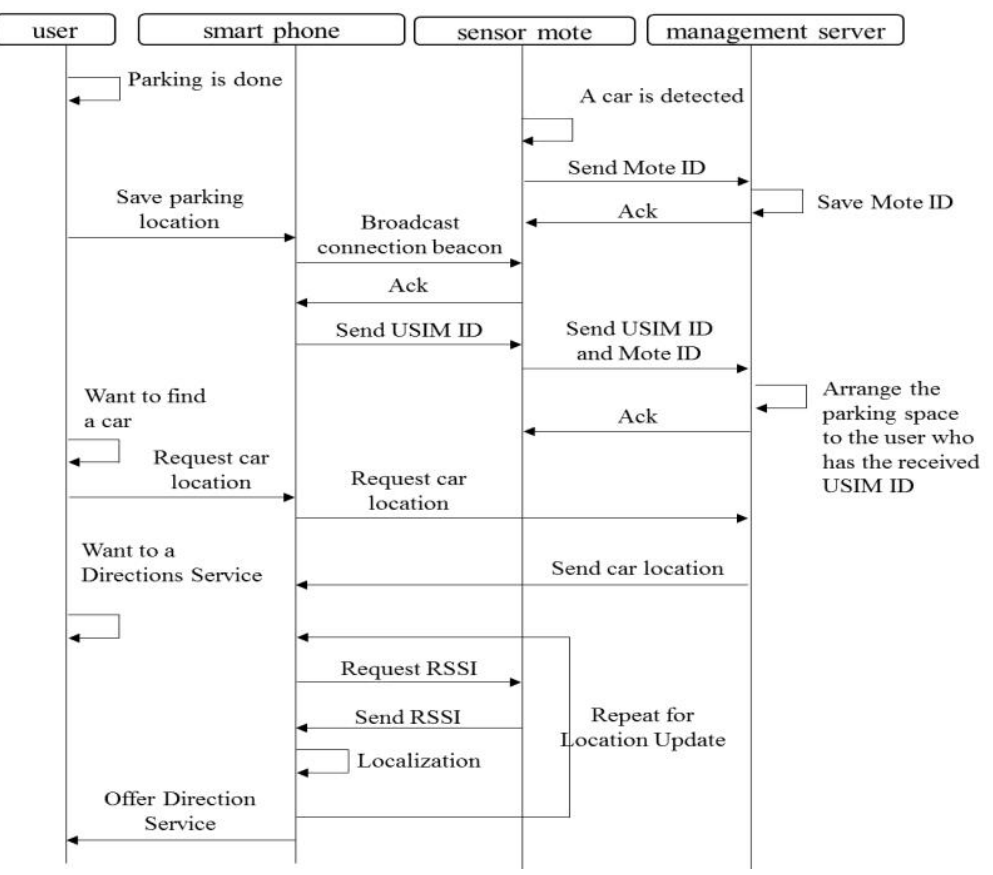

Fig. 6: Schematic illustrating the smart parking system.

\subsection{Smart Parking Location Guidance Service}

The $A^{*}$ algorithm is used to provide the location guidance service from the user's current location to the parked vehicle. The $\mathrm{A}^{*}$ algorithm is used to find the shortest path from the start point to the destination point, where the method employed is simple but it performs well (Hart et al. 1968). To apply this algorithm, it is necessary to classify the movable cells and unmovable cells in the parking area. In our method, the occupied parking spaces and walls in the parking area are regarded as unmovable cells. The empty parking spaces and the paths of vehicles or people are classified as movable cells. 


\subsection{Operation of the Smart Parking System}

Figure 6 illustrates the smart parking system. After the user finishes parking their vehicle, the sensor mote above the parking space transmits the mote ID to the management server. The management server updates the parking state for the corresponding space. Next, the user executes the parking application on their smartphone to save the location of their parked vehicle. The parking application employs the RSSI of the BLE module to recognize the location of the parked vehicle. The USIM ID of the user's smartphone is then sent to the sensor mote before the sensor mote transmits the received USIM ID and the mote ID to the management server. The management server saves all the information.

When users request parking location information regarding their vehicles, the parking application submits the USIM ID to the management server. In addition, if the user requires the location guidance service to find their parking space, the parking application periodically receives RSSI data from the sensor motes deployed around the user. The RSSI data are then transformed into the distances between the user and the sensor motes. Based on three selected distances, the triangulation method is applied periodically and the current location of the user is recognized so the location guidance service can be provided in real-time.

\section{Implementation and Experiments}

\subsection{Parking Sensor Mote}

As shown in Figure 7, we implemented the parking sensor mote proposed in this study, which comprised a Devantech SRF04 ultrasonic sensor, Jinan Huamao HM10 BLE module, and Crossbow MicaZ control module. Table 1 shows the specifications of the MicaZ mote. The MicaZ is based on an Atmega 128L processor with low power consumption, which has high scalability features with 51 extension pins. The MicaZ has been employed previously in implementations of wireless sensor networks in the IoT because of these features (Online). In this study, we used TinyOS as middleware for the sensor mote, which is based on an eventbased operating system and it supports low power operation (Online).

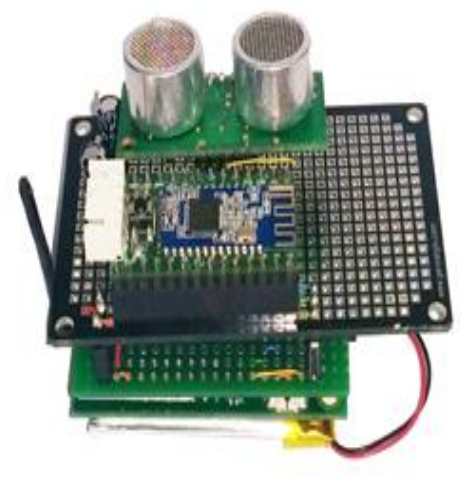

Fig. 7: Parking sensor mote. 
Table 1: Specifications of the Micaz mote

\begin{tabular}{|l|l|}
\hline MCU & ATMEGA 128L 8 Mhz \\
\hline Memory & 4 K RAM/128 K FLASH \\
\hline I/O and Sensors & Large expansion 51-pin connector \\
\hline & $\begin{array}{l}\text { Frequency band }=2.4 \mathrm{GHz} \\
\text { Data transmission rate }=250 \mathrm{Kbps} \\
\text { RF Transceiver }\end{array}$ \\
& $\begin{array}{l}\text { RF power }=-24 \mathrm{dBm} \text { to } 0 \mathrm{dBm} \\
\text { Indoor range }=20 \mathrm{~m} \text { to } 30 \mathrm{~m}\end{array}$ \\
\hline
\end{tabular}

\subsection{User Parking Application}

The user parking application was developed in Android 4.1 version and installed on a smartphone. The basic screen displayed the current state of the parking space and it indicated the location where the user's vehicle was parked. It also included functions for saving the location of the parked vehicle and the location guidance service to parking spaces was provided.

Figure 8 shows the interface screen for the user parking application. The cells represent parking spaces or the pathways for vehicles and pedestrians. The gray cells indicate the available parking spaces and the black cells are the parking spaces occupied by vehicles. The white cells represent the pathways for vehicles and people.

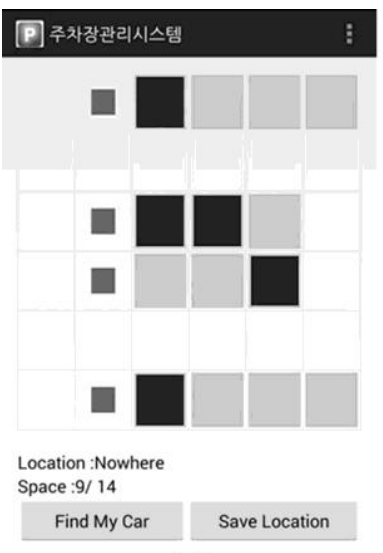

(a)

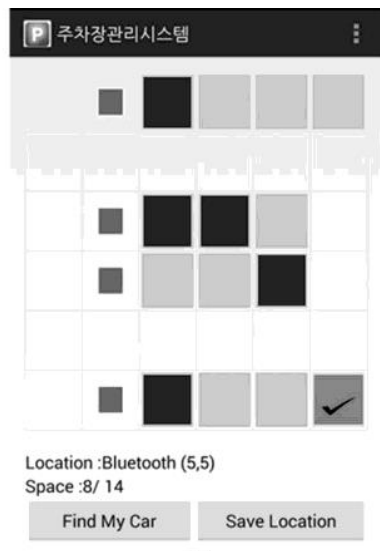

(b)

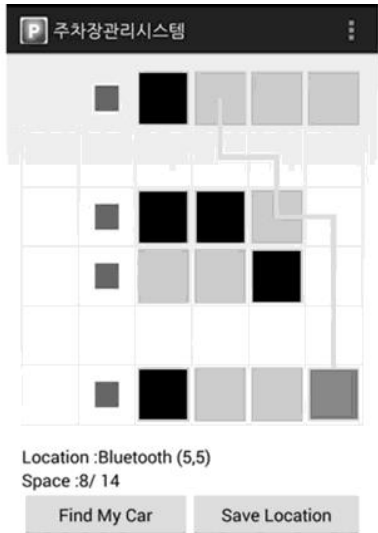

(c)

Fig. 8: User parking application: (a) saving the parking location, (b) current state of parking spaces, and (c) location guidance service.

The user interface is classified according to three types. As shown in Figure 8(a), the first interface shows the operation of the function for saving the current location of the parked vehicle. If the user touches the "Save Location" button, the parking application begins to communicate with the sensor mote above where the vehicle is parked. The USIM ID is sent for user identification to the sensor mote. The sensor mote transmits the location information for the parked vehicle and the user ID to the server. The second interface displays the current state of the parking 
spaces. As shown in Figure 8(b), a vehicle begins to occupy a parking space indicated by the bottom line of the grid. The color of the cell changes from a dark gray color to a checkmark. All of the information related to the parking spaces and the location of the user's vehicle can be monitored via this interface. The third interface provides the location guidance service to parking spaces. As shown in Figure 8(c), if the user touches the "Find My Car" button, real-time direction guidance service is provided based on the triangulation method by using the RSSIs of the BLE until the user reaches their parked vehicle. The lines in Figure 8(c) indicate the direction in which the user is guided.

\subsection{RSSI Strength of Sensor Motes}

In this study, the BLE modules of the sensor motes were controlled at four signal strength levels, i.e., $-23 \mathrm{dBm},-6 \mathrm{dBm}, 0 \mathrm{dBm}$, and $6 \mathrm{dBm}$. The RSSI data indicated the different values according to the strength of each signal. In order to save the locations of parked vehicles in a reliable manner, we performed an experiment to determine the optimal signal strength within a range of 2-3 $\mathrm{m}$. As mentioned in Section 3.1, this is the distance between the sensor mote on the ceiling and the floor of the parking space.

Figure 9 shows the experimental environment used to measure the signal strengths from the BLE module, where the parking sensor mote was at a height of 2 $\mathrm{m}$ and we measured the signal strengths. The measurements were performed 300 times.

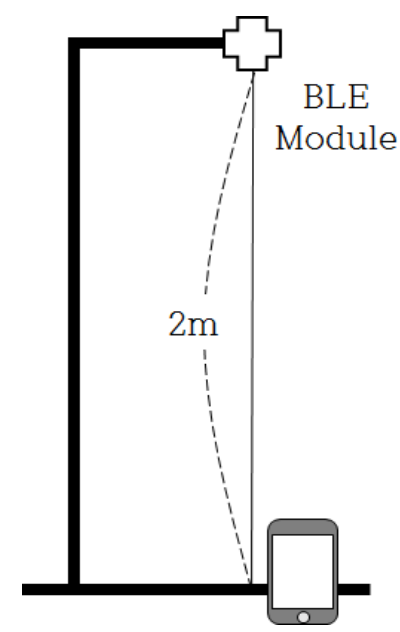

Fig. 9: Experimental environment for determining the RSSI strength of sensor motes.

Figure 10 shows the RSSI values achieved with the BLE module at four signal strengths. At $2 \mathrm{~m}$, the measured RSSI value was not proportional to the signal strength for the BLE module. According to the signal strength, the RSSI values exhibited different characteristics. Thus, at $-23 \mathrm{dBm}$, the signal strength was strong and the fluctuations in the RSSI range were not large. At $-6 \mathrm{dBm}$, the signal 
strength was low and the fluctuations in the RSSI range were similar to those at -23 $\mathrm{dBM}$. However, at $0 \mathrm{dBm}$, the variation in strength was almost the same as that at $6 \mathrm{dBm}$, but the fluctuations in the RSSI range were large. The fluctuations in the RSSI range were excessively high at $6 \mathrm{dBm}$.
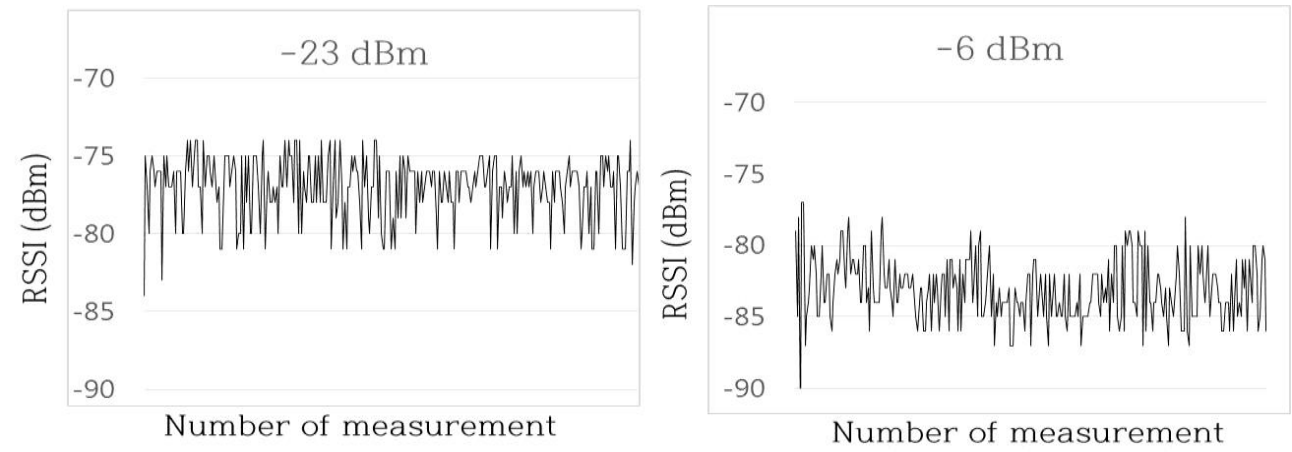

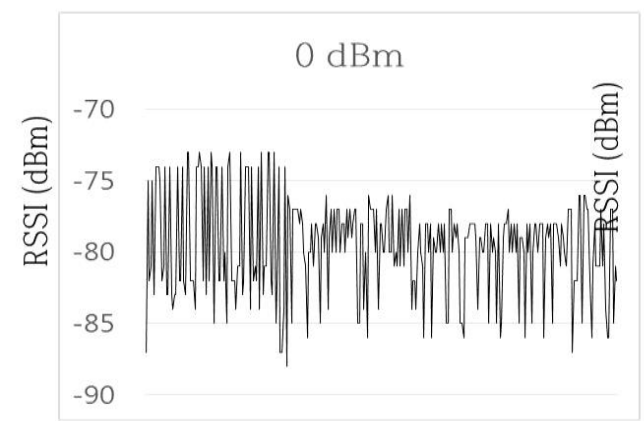

Number of measurement

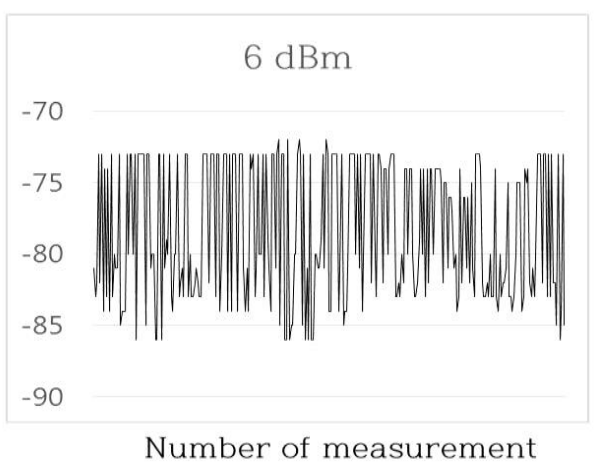

Fig. 10: Fluctuations in the RSSI range with different BLE signal strengths.

Thus, to improve the recognition rate for parked vehicles, we recommend that the signal strength is weak and that the range of fluctuations in the RSSI is small. If the RSSI strength is strong, then many sensor motes will be found by the user's smartphone, so the speed of measurement will be delayed and there may be difficult selecting the correct sensor motes. In addition, high fluctuations in the RSSI range may also make it difficult to select appropriate sensor motes, thereby leading to unreliability. Thus, we selected a signal strength of $-6 \mathrm{dBm}$ because it produced small fluctuations and the signal strength was weak.

\subsection{Sensor Mote Selection for Parked Vehicles}

Several sensor motes may be connected with a user's smartphone, but the sensor mote with the strongest RSSI should be selected before saving the location of the parked vehicle. Next, we constructed an experimental environment according to the specifications for a real parking space in order to evaluate the accuracy of the selection process. As shown in Figure 11, three sensor motes were deployed, where the distances between sensor motes were $2.3 \mathrm{~m}$ and that from the sensor mote to the 
smartphone was kept at $2.6 \mathrm{~m}$. These distances were based on real parking spaces. Each space was occupied by a vehicle. We measured the RSSI for sensor motes located above each parking space. The accuracy evaluation confirmed a parked vehicle based on the sensor mote with the highest signal strength.

In the experiments, we collected 1-8 RSSI data during each one minute interval for each parking space. The average values were calculated to increase accuracy. In total, 100 accuracy evaluations were performed.

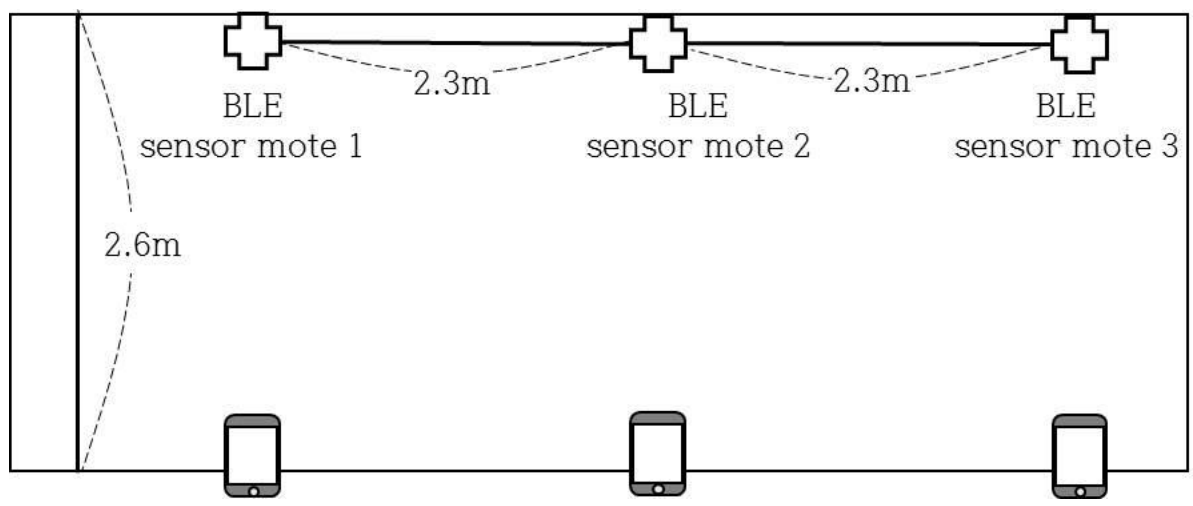

Fig. 11: Experimental environment employed for sensor mote selection.

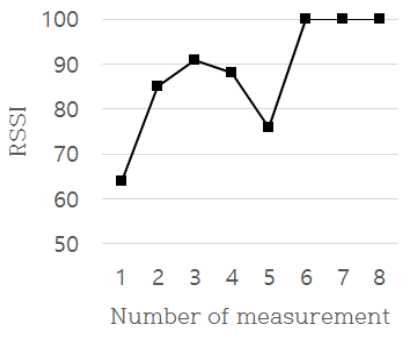

(a)

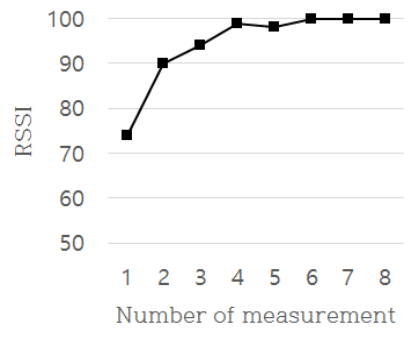

(b)

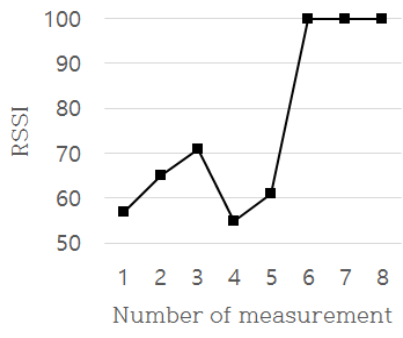

(c)

Fig. 12: Accuracy of sensor mote selection: (a) sensor mote 1, (b) sensor mote 2, and (c) sensor mote 3 .

Figure 12 shows the accuracy obtained when the sensor mote with the highest RSSI was selected, where the X-axis denotes the number of RSSI measurements in each one minute interval and the $\mathrm{Y}$-axis represents the probability of choosing the correct sensor mote. The correct sensor mote was assigned to the parking space where the vehicle was parked. As shown in Figure 12, the probability was almost $100 \%$ when over six measurements were made. However, the time required to obtain the measurements could be long when the number of measurements exceeded six. Thus, we made three measurements, which were completed in 1-2 seconds, and the average accuracy was $83 \%$ according to our experiment.

In our smart parking system, the sensor motes with the highest signal strength are in sequence, so the states of the connected sensor motes can be confirmed in 
real-time. Therefore, a difference in the search time exists over a small range, but the sensor mote corresponding to the parked vehicle is selected accurately.

\subsection{Evaluation of the Distance Equation for Location Awareness}

A location awareness method that helps users find their parking space is needed in order to provide the location guidance service mentioned in Section 3.3. Thus, we derived the distance estimation equation (Equation (2)). However, the distances obtained from the equation are estimated values. Thus, we evaluated the accuracy of Equation (2) by comparing the estimated distances with the actual distances between the sensor mote and the user's smartphone. In this evaluation, we defined the error range as the difference between the real distance and the estimated distance.

In this experiment, we measured the RSSI strengths while the user's smartphone moved from the sensor mote to a maximum distance of $5 \mathrm{~m}$. The measurements were performed at $10-\mathrm{cm}$ intervals over $5 \mathrm{~m}$. The measurements were obtained 10 times in the same environment and the average values were used in the evaluation. Furthermore, after excluding the minimum and maximum values, the average values were also calculated to enhance accuracy. Finally, the error ranges were obtained by comparing the values estimated by Equation (2) and the real measured values.

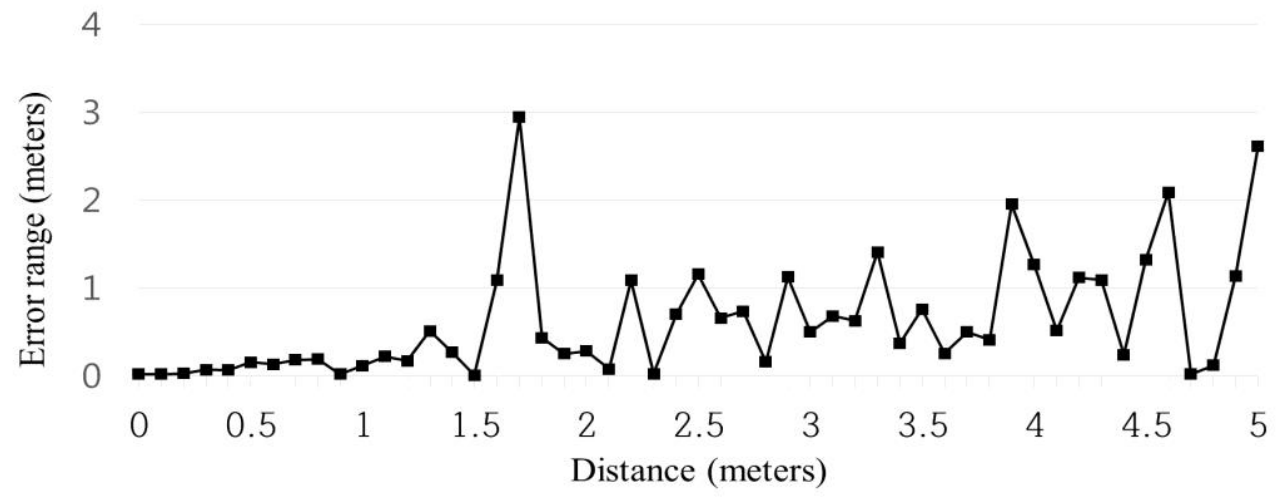

Fig. 13: Error ranges between the real and estimated distances.

Figure 13 shows the error ranges between the real distances and the distances estimated by Equation (2). At less than $2.3 \mathrm{~m}$, the error was $50 \mathrm{~cm}$, which indicates the high reliability of this method for location awareness. However, up to $5 \mathrm{~m}$, the errors were about $25 \%$. The maximum and minimum values were excluded to enhance the accuracy, and the error ranges are shown in Figure 14, which indicates that the error decreased to $22 \%$. Thus, although errors existed, the error was below 1 $\mathrm{m}$ at up to $5 \mathrm{~m}$ and the maximum error was below $2 \mathrm{~m}$. A cell-based location awareness method is employed in our smart parking system, so the values estimated by Equation (2) can be used to support the location guidance service. Finally, we 
found that there was no difficulty searching for the parking space using this method.

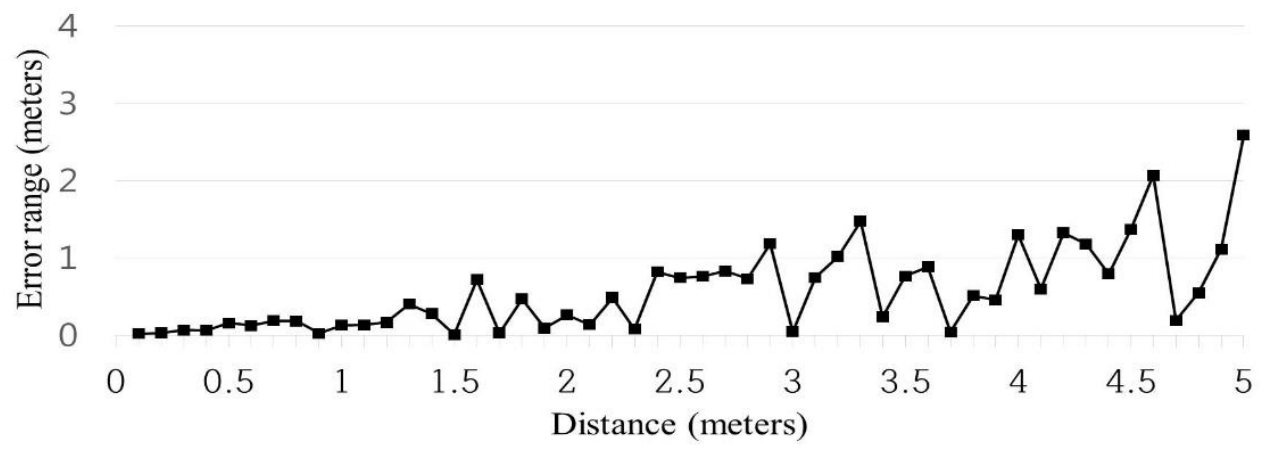

Fig. 14: Error ranges without the maximum and minimum values.

\section{Conclusion}

In this study, we implemented a smart parking system based on sensor motes with ultrasonic sensors and BLE communication devices in the IoT. The states of the parking spaces are checked in real-time by the smart parking system. In addition, based on user location awareness technology, the locations of parked vehicles are recognized and a location guidance service is provided to help users find their parking space.

The ultrasonic sensor in the sensor mote is employed to determine the states of parking spaces. The ultrasonic sensor calculates the distance between the sensor mote and the floor of the parking space using a backward echo ultrasonic wave. If the distance between the sensor and the floor is below $100 \mathrm{~cm}$, then the space is regarded as being occupied by a vehicle. We also implemented two location awareness methods based on the RSSI of the BLE modules in sensor motes. In order to obtain reliable RSSI data, we measured the fluctuations in the RSSI range according to the signal strength of the BLE module. We found that reliable RSSI data could be received when the signal strength was weak and the fluctuations in the RSSI were small. Therefore, we decided to use a signal strength of $6 \mathrm{dBm}$ in our experiment.

When three measurements of RSSI data were obtained in one interval, the accuracy of sensor mote selection was $83 \%$ for the sensor mote related to the parked vehicle. In our experiment, the selection of the correct sensor mote required slightly more search time, but the proposed method found the sensor mote accurately within 1-2 seconds. Thus, we developed a location awareness method for saving the locations of current parked vehicles based on the accurate selection of sensor motes.

We also implemented another location awareness method to guide users to the location of their parking space, where we derived an equation for transforming the RSSI strength into the distance. This equation estimates the distances between the 
user and three sensor motes. Based on these three distances, the triangulation method is employed to provide location awareness and guide the movements of a user to their parking space. According to our experiments, the equation made some errors when calculating the distance but the parking spaces were assigned to cell units, so the proposed method successfully supported the location guidance service without difficulty.

In our future research, we will study the effective reservation of parking spaces and an alarm system for detecting vehicle accidents or for maintaining security in smart parking spaces. The accuracy of location awareness will be enhanced for this purpose. Moreover, the multimodal interactions between other sensors in addition to the BLE will be investigated to facilitate detailed location awareness in offices or home spaces.

\section{References}

Crossbow from http://xbow.com

Gilbert, E. P. K., Kaliaperumal B. \& Rajsingh E. B. (2012). Research issues in wireless sensor network applications: a survey. International Journal of Information and Electronics Engineering, 2, 702-706.

Gomez, C., Oller, J. \& Paradells, J. (2012). Overview and evaluation of bluetooth low energy: An emerging low-power wireless technology. Sensors, 12, 1173411753.

Han, S., Choi, T., Ryu, D. \& Shin, S. (2011). Error Compensation Algorithm of CSS-Based Real-Time Location Awareness Systems. The journal of the Institute of Internet Broadcasting and Communication, 11, 119-126.

Hart, P. E., Nilsson, N. J. \& Raphael, B. (1968). A formal basis for the heuristic determination of minimum cost paths. Systems Science and Cybernetics, IEEE Transactions on, 4, 100-107.

Jeon, S., Kwon, E. \& Jung, I. (2014) Traffic Measurement on Multiple Drive Lanes with Wireless Ultrasonic Sensors. Sensors, 14, 22891-22906.

Jin, J., Gubbi, J., Marusic, S. \& Palaniswami, M. ( 2014). An information framework for creating a smart city through Internet of things. Internet of Things Journal IEEE, 1, 112-121.

Jo, Y., Choi, J. \& Jung, I. (2014). Traffic Information Acquisition System with Ultrasonic Sensors in Wireless Sensor Networks. International Journal of Distributed Sensor Networks, 1, 1-12.

Jung, G. \& Sim, K. (2010). Mutual Localization of swarm robot using Particle Filter. Journal of Korean Institute of Intelligent Systems, 20, 298-303. 
Kaune, R., Horst, J.\& Koch, W. (2011) Accuracy Analysis for TDOA Localization in Sensor Networks. 14th Int. Conf. on Information Fusion, 1-8.

Lee, H., Kim, D., Kim, D., \& Bang, S. (2003). Real-time automatic vehicle management system using vehicle tracking and car plate number identification. International Conference on Multimedia and Expo, 2, 353-356.

Mainetti, L., Palano, L., Patrono, L., Stefanizzi, M. L. \& Vergallo, R. (2014). Integration of RFID and WSN technologies in a Smart Parking System. 22nd International Conference on Software, Telecommunications and Computer Networks (SoftCOM), 104-110.

Sathyan, T., Hedley, M.\& Mallick, M. (2010) An analysis of the Error Characteristics of Two Time of Arrival Localization Techniques. 13th International Conference on Information Fusion, 1-7.

TinyOS from http://tinyos.net/

Wei, L., Wu, Q., Yang, M., Ding, W., Li, B. \& Gao, R. (2012). Design and Implementation of Smart Parking Management System Based on RFID and Internet. 2012 International Conference on Control Engineering and Communication Technology, 17, 7-9.

Zaruba, G. V., Huber, M., Kamangar, F. A. \& Chlamtac, I. (2007). Indoor location tracking using RSSI readings from a single Wi-Fi access point. Wireless Networks, $13,221-235$. 